making timely screening imperative for infection control. While enough evidence exists on diagnostic accuracy measures for pointof-care tests (POCTs), the quality of evidence on measures beyond accuracy is poor. We reviewed evidence on these implementation research outcomes (IROs) and summarised their quality.

Method Two reviewers systematically searched $10+$ electronic databases for the period: January 1980-September 2012, independently abstracted data and synthesised outcomes narratively. Over 10,000 citations were screened and a final set of 191 studies identified for inclusion.

Results Of 191 studies, almost half $46 \%(\mathrm{n}=127)$ in HIV and $41 \%$ $(n=64)$ in syphilis, reported IROs. IROs included acceptability, preference, feasibility and impact. Across 16 studies, acceptability measure was reported as proportions, rates, without confidence intervals often without clear definitions. Across 9 studies, preference was reported as proportion, without definitions or comparators. Feasibility metric across 7 studies, was ill-defined and heterogeneously reported as either completion of strategy, or test procedure, often as a statistic without confidence intervals or a definition or a quantifiable metric. Impact measure $(\mathrm{n}=13)$ was best quantified in clinical trials-reported as either time to treatment initiation, or time to receiving a test result, or change in numbers newly infected or screened with a POCT strategy. Unclear definitions of other IROs, lax measurement resulted in deficient documentation and weak quality ratings on STROBE and CONSORT checklists, raising concerns about the quality of the evidence presented.

Conclusion Poor reporting of IROs (i.e, feasibility, acceptability, preference) in POCT diagnostics masked evidence and pointed to the need for standardised definitions, quantification and reporting for them. A framework for documenting metrics beyond accuracy and impact is urgently needed to improve evaluation of true benefits of POCT diagnostics in implementation research.

\section{P3.323 PREVALENCE OF CHLAMYDIA TRACHOMATIS IN THE UNITED STATES AFTER ADJUSTING FOR SENSITIVITY AND SPECIFICITY OF THE SCREENING TEST}

doi:10.1136/sextrans-2013-051184.0776

\section{A Hadgu. Centers forDiseaseControl and Prevention, Atlanta, GA, United States}

According to the Centers for Disease Control and Prevention (CDC), Chlamydia trachomatis infection is among the most prevalent of all sexually transmitted diseases (STDs), and since 1994, has comprised the largest proportion of all STDs reported to CDC. In the past, researchers have used nationally representative surveys, such as, the National Health and Nutrition Examination Survey to estimate chlamydia prevalence and trend under the assumption that the test used to screen for chlamydia has perfect sensitivity and specificity. Under such assumption, the prevalence of chlamydial infection in the U.S. is $2.2 \%$ (CI, $1.8 \%$ to $2.8 \%$ ). However, chlamydia screening tests are not perfect tests and thus prevalence estimates must account and adjust for these imperfections. Statisticians have shown that estimates of disease prevalence based on the assumption that screening tests have perfect sensitivity and specificity can be severely biassed. In this work, we use Bayesian methods to provide sensitivity and specificity adjusted estimates of chlamydia prevalence. Based on this method, the adjusted prevalence estimate of chlamydia in the U.S. is $1.1 \%$ (CI, $0.002 \%$ to $2.02 \%$ ).

\section{P3.324 A POPULATION-BASED ASSESSMENT OF RACIAL/ETHNIC DISPARITIES IN GONORRHOEA RATES}

doi:10.1136/sextrans-2013-051184.0777

${ }^{1} Y$ Ransome, ${ }^{2} \mathrm{D}$ Nash. ${ }^{1}$ Columbia University Mailman School of Public Health, New York, NY, United States; ${ }^{2}$ CUNY School of Public Health at Hunter College, New York, NY, United States
Background Measured incidence rates of Sexually Transmitted Infections (STIs) are substantially higher among blacks compared to whites in the US. However, population-based data sources, such as routine STI surveillance systems, often lack information on race/ ethnicity for the majority of cases. We compared black-white differences in gonorrhoea rates using both individual and neighbourhoodlevel measures of race/ethnicity.

Methods Publicly-available aggregate data on the number of New York City(NYC) adult gonorrhoea cases in 2009 by race/ethnicity and sex were obtained from the NYC Department of Health and Mental Hygiene's online system for NYC's 42 neighbourhoods. The proportion of black residents for each neighbourhood was obtained from census data.

Results The citywide gonorrhoea rate in 2009 was 81 per 100,000 (median for 42 neighbourhoods [median]: 29; intraquartile range across 42 neighbourhoods [IOR]: 2-105); 116 per 100,000 for males (median:44; IOR: 11-177); and 47 per 100,000 for females (median: 10; IOR: 0-67). Race/ethnicity data were missing for $49 \%$ of cases (median: 48\%; IOR: 36\%-61\%). Using data on cases with complete information on race/ethnicity, gonorrhoea rates were 225 per 100,000 (median: 204; IQR: 108-321) among non-Hispanic blacks, compared with 33 per 100,000 (median: 6; IQR: 1-37) among nonHispanic whites. The median black-white difference in gonorrhoea diagnosis rates was 104 per 100,000, and varied substantially across neighbourhoods (IOR: 23-223). Neighborhoods in the lowest quartile of the proportion of black residents (where $0.1 \%-0.4 \%$ of neighbourhood residents were black) had the lowest mean gonorrhoea rate 33 per 100,000, compared to 81,162 , and 224 in the $2^{\text {nd }}, 3^{\text {rd }}$ and $4^{\text {th }}$ quartiles (where $35 \%-76 \%$ of neighbourhood residents were black), respectively $(\mathrm{p}<0.001)$.

Conclusions Analyses using individual-level and neighbourhoodlevel race/ethnicity measures both suggest substantial black-white differences within and across NYC neighbourhoods. More complete race/ethnicity information among persons diagnosed with gonorrhoea is critical to elucidate possible structural/neighbourhood determinants of black-white differences.

\section{P3.325 EVALUATING CONSISTENCY IN REPEAT SURVEYS OF MEN WHO HAVE SEX WITH MEN (MSM) USING RESPONDENT- DRIVEN SAMPLING IN ZANZIBAR ISLAND, ZANZIBAR - TANZANIA}

doi:10.1136/sextrans-2013-051184.0778

'S Haji, ${ }^{1} \mathrm{~A}$ Khatib, ${ }^{1} \mathrm{M}$ A Khamis, ${ }^{2} \mathrm{C}$ Said, ${ }^{3} \mathrm{E}$ Matiko, ${ }^{1} \mathrm{~F}$ Khalid, ${ }^{4} \mathrm{M}$ Dahoma, ${ }^{1} \mathrm{~A}$ Ali, ${ }^{1} \mathrm{~A}$ Othman, ${ }^{3} \mathrm{M}$ Kibona. 'Zanzibar AIDS Control Programme, Ministry of Health, Zanzibar, Tanzania; ${ }^{2}$ Global Health Sciences, University of California, San Francisco, CA, United States; ${ }^{3}$ Division of Global HIVIAIDS, US Centers for Disease Control and Prevention, Dar es Salaam, Tanzania; ${ }^{4}$ Directorate of Preventive Services and Health Education, Ministry of Health, Zanzibar, Tanzania

Background This study assessed the comparability of respondentdriven sampling (RDS) as a sampling and recruitment method by comparing two cross-sectional surveys conducted among MSM in Zanzibar using RDS in 2007 and 2011.

Methods We conducted community-based behavioural surveillance studies in Zanzibar using respondent-driven sampling (RDS) to recruit 509 MSM in 2007 and 344 in 2011. We used crude and RDSAT-adjusted descriptive statistics to assess differences between the samples.

Results Compared to 2007, participants in 2011 were significantly younger (31.4\% vs $9.9 \%$ were younger than 19 , p < 0.001 ), more likely to have been tested for HIV in the last year $(53.7 \%$ vs $10.6 \%$, $\mathrm{p}<0.001)$, ever tested $(68.2 \%$ vs $18.8 \%, \mathrm{p}<0.001)$, and less likely to have injected drugs in the last 3 months $(1.0 \%$ vs $23.2 \%, p<0.001)$. In 2011, 12 (2.6\%) tested positive for HIV; in 2007, 65 (12.3\%) were positive $(p<0.001)$. 